\title{
Beauty is only partly in the eye of the beholder
}

Bird Coloration, Volumes I,II edited by Geoffrey E. Hill and Kevin J. McGraw. Harvard University Press, 2006. Each £61.95, hbk (589 and 528 pages) ISBN 0674018931/0674021762

\section{Phil F. Battley and Mark E. Hauber}

\author{
School of Biological Sciences, University of Auckland, PB 92019, Auckland, New Zealand
}

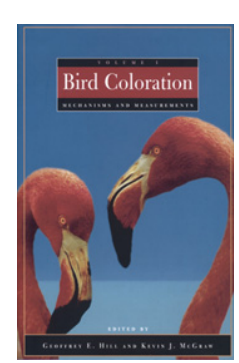

The splendid colours and complex patterns of the plumage, eggs, and beaks, feet and other bare parts of birds have captivated humans for millenia and scientists for more than 150 years. In particular, birds have provided a rich basis for mechanistic studies of feather structure and plumage development, predominant through much of the 20th century, and for evolutionary studies of functional aspects

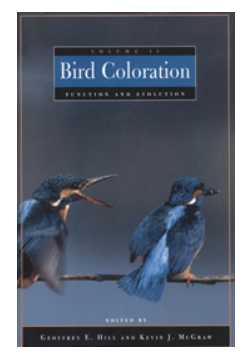
of the plumage, which began with Darwin and Wallace but followed by a hiatus until the latter half of the 20th century. The past three decades have given rise to a renewed and prolific research field that combines mechanistic and evolutionary approaches to the study of the colour of birds and Avian Coloration, edited by Geoffrey Hill and Kevin McGraw, provides a state-of-the-art review of the field as it currently stands. Here, 22 specialists provide syntheses of key topics, split for convenience across two volumes.

Volume I, Mechanisms and Measurements, covers three main areas: perception and measurements, mechanisms of production, and control and regulation of expression. The first section introduces the fundamental issue that human vision differs from avian vision. Although much human fascination with birds stems from our shared major senses used in communication (vision and hearing), the ability of birds to detect ultraviolet wavelengths means that there is a hidden world of signaling that scientists have only recently been able to address. This has now changed with the development of portable spectrometers, and instead of denoting colours we now measure reflectance spectra. The opening chapters on quantifying and analysing colours provide a thorough theoretical and practical background to this technique, down to issues as seemingly trivial as appropriate naming of computer files (but which can cause headaches otherwise). Although the beauty of reflectance is that it truly is an objective property of the subject under scrutiny and, hence, independent of our own visual and cognitive biases, it is still only one step closer to representing the information perceived by birds themselves. Additional physiological or neural filters in avian sensory and recognition systems, as well as environmental factors, affect what birds actually 'see', a point developed early in the book.

Corresponding author: Battley, P.F. (pbat014@ec.auckland.ac.nz).

Available online $\mathrm{xxxxxx}$.
The second section of Part $I$ is the most reference-book-like and comprehensive chapters cover the mechanics of production of carotenoids, melanins, structural colours, pterins, porphyrins and psittacofulvins, as well as an engaging account of cosmetic and adventitious colours. As carotenoids have featured so strongly in the study of avian signals, it is not surprising that this chapter is the longest; however, there is equally detailed treatment of the commonest of avian pigments, melanins, which makes a strong case that they are far more interesting than is generally accepted. A salutary message here is the need to identify formally the pigments involved in colouration. Also, anyone working on birds that happen not to incorporate any carotenoids into feathers need no longer feel left out.

The final section of Part I covers the regulation of colouration. Hormonal regulation, although well studied, is not comprehensively understood, in part because of difficulties in controlling for secondary changes in experimental treatments. Genetic factors are comparatively poorly studied, but the recent discovery that variation in the gene encoding the melanocortin-1 receptor corresponds with variation in melanism in three polymorphic species [1] provides a model for future work. Some (speculative) discussion on how melanism might be controlled in predominantly dark juveniles of the otherwise pale-morph of one of those species, the Arctic skua Stercorarius parasiticus, would have been welcome here. Environmental effects on the expression of colours are discussed thoroughly, particularly of carotenoid-based colours.

Volume II, Function and Evolution, contains ten essays, seven on function, or the current utility, of colouration and just three on evolutionary aspects and transitions of colour states. The former focus on the selective forces that shape colour diversity across species, including natural selection, sexual selection and 'social' selection. Within the chapters on function, most authors follow a traditional scientific approach by first reviewing what the structural basis is of different avian colours and how to quantify plumage and skin colouration, thereby providing a strong link with Volume I. This is accompanied by addressing what phenotypic traits, including dominance and parental traits, correlate with variation in colour, and what experimental results support the potential signaling- and social discrimination-related functions of bright or cryptic colouration. Comparative methods are extensively used in just two functional chapters, regarding the diverse types and possible selective advantages of colourful chick and female phenotypes, despite two of the three evolutionary chapters 
advocating the use of the comparative method to test ecological and functional hypotheses of avian colour diversity. These latter two chapters on evolution contrast somewhat with the approach of the third (phenotypes versus genotypes), in which trajectories of colour diversity are placed within the concept of modularity, developed in the framework of evolutionary-developmental arguments [2].

A chapter on intraspecific variation in colour also provides several previously unpublished plots and figures, suggesting that polymorphisms within taxa are a worthwhile and productive research avenue. The other chapters in Volume II are also exciting treasure-troves of extensive tabulated alternative hypotheses, lists and details of observational and experimental data across a multitude of species, and generously broad sets of interpretation of these results. Each of these tables could well be the fruitful basis for further analyses using the comparative methods described in final chapters of the volume. Perhaps it might have been helpful to plot experimental data on specific tests of functional hypothesis against a biogeographical map of species diversity or a phylogenetic distribution of colour-trait diversity to highlight species, lineages and habitats for which crucial data are missing from the published literature.

Both volumes have a non-paginated set of colour plates to provide examples from each chapter. In contrast to the beautiful cover photos, plates in Volume I are oversaturated and Volume II are undersaturated, but they nevertheless provide impressive illustrations of colouration and colour diversity within and across species (except the mislabelling of the photo of the parasitic brown-headed cowbird Molothrus ater egg that has gray catbird eggs Dumetella carolinensis for the host). It would have been useful to have more examples of standard techniques of photographing and measuring plumage and skin colouration, such as those used to photography common murre Uria aalge eggs or the gape of the red-throated parrotfinch Erythrura psittacea chick.

Avian Coloration is a masterly compilation that is an essential reference for anyone contemplating work on the subject. It combines the right mix of theoretical reasons for studying colouration and the practical information needed to do so, and repeatedly tabulates and presents testable ideas for future projects. In an era when 'multidisciplinary' is a university catchword but which can be difficult to achieve, Avian Coloration provides a template for bringing together behavioural ecologists, evolutionary biologists, biochemists, anatomists and geneticists to understand the origins and functions of avian colour. The techniques are now available to measure objectively the basis of many colours and their visual properties. Although beauty to us might be in the eyes of the beholder, researchers are now better able to overcome our visual limitations and measure what might really be relevant to birds. This book will serve as an important stimulus in this direction and promises an exciting future for the study of colouration in birds and, perhaps, other taxa.

\section{References}

1 Mundy, N.I. (2005) A window on the genetics of evolution: $M C 1 R$ and plumage colouration in birds. Proc. R. Soc. B 272, 16331640

2 West-Eberhard, M.J. (2003) Developmental Plasticity and Evolution, Oxford University Press 